JOURNAL OF THEORETICAL

AND APPLIED MECHANICS

53, 3, pp. 643-652, Warsaw 2015

DOI: $10.15632 /$ jtam-pl.53.3.643

\title{
THE COMBINED HEAT TRANSFER OF RADIATION AND MIXED CONVECTION ANALYSIS IN A LID-DRIVEN TRAPEZOIDAL CAVITY
}

\author{
Malihe Mohammadi, Seyyed Abdolreza Gandjalikhan Nassab \\ Department of Mechanical Engineering, School of Engineering, Shahid Bahonar University, Kerman, Iran \\ e-mail:mohammadi2345@yahoo.com
}

\begin{abstract}
In this paper, the effect of radiation heat transfer on mixed convection in a lid-driven trapezoidal cavity is studied numerically. The governing equations of mixed convection are solved based on the SIMPLE algorithm and the solution of the radiative transfer equation inside the participating medium is carried out using the discrete ordinates method, simultaneously. To study the heat transfer and flow characteristics, sensitivity analysis is carried out based on the two parameters including the optical thickness and radiation-conduction parameter. Several interesting results are obtained such as sweep behavior on the isotherms, streamlines and convective Nusselt number with optical thicknesses.
\end{abstract}

Keywords: mixed convection, radiation, lid-driven trapezoidal cavity

\section{Introduction}

The mixed convection process inside a lid-driven cavity is found in many engineering applications such as solar collectors, cooling of electronic devices, heat exchangers (Cheng and Liu, 2010). Study of the literature shows that the problem is considered along two different parts, those are square or rectangular geometry and irregular geometries. The majority of previous works investigated the effect of several boundary conditions on flow and temperature characteristics inside a lid-driven square or rectangular cavity (Moallemi and Jang, 1992; Prasad and Koseff, 1996; Shankar et al., 2002; Oztop, 2006). Chen and Cheng $(2004,2005)$ studied the flow and temperature characteristics for mixed convective inside an arc-shaped lid-driven cavity by considering variable inclination angle. Recently, few literatures items have been reported on topic of mixed convective flow inside a lid-driven trapezoidal cavity. The analysis of flow and temperature characteristics inside irregular geometries is very important for advance of heating and cooling arrangements or material processing systems (Bhattacharya et al., 2013). In most of the studies on the mixed convection in lid-driven cavities, the role of the radiative heat transfer is neglected. Therefore, the objective of this work is to study the effect of radiative heat transfer on mixed convection in a lid-driven trapezoidal cavity numerically. To achieve this purpose, the flow and heat transfer characteristics is investigated for a broad range of optical thickness $(0<\tau<100)$ and radiation-conduction parameters $(0.5<R C<10)$ at the Richardson number equal to 10 . The lid-driven trapezoidal cavity is considered as emitting, absorbing and isotropically scattering participating with gray gasses. The governing equations are solved by using the finite volume method and the radiative transfer equation by the discrete ordinates method (DOM). Also, the SIMPLE algorithm of Patankar and Spalding (1972) has been employed to couple the velocity and pressure fields. It should be mentioned that analysis of combined radiative heat transfer and mixed convection in a lid-driven trapezoidal cavity is carried out for the first time in the present work. 


\section{Nomenclature}

$C_{p} \quad-\quad$ specific heat $[\mathrm{J} /(\mathrm{kg} \cdot \mathrm{K})]$

$g \quad-$ gravitational acceleration $\left[\mathrm{m} / \mathrm{s}^{2}\right]$

$I \quad-$ radiation intensity $\left[\mathrm{W} / \mathrm{m}^{2}\right]$

$I^{*} \quad-$ dimensionless radiation intensity

$k \quad-\quad$ thermal conductivity $[\mathrm{W} /(\mathrm{m} \cdot \mathrm{K})]$

$L \quad$ - the enclosure height $[\mathrm{m}]$

$p \quad$ - fluid dynamic pressure $[\mathrm{Pa}]$

$P \quad-$ dimensionless pressure

$q_{c}, q_{r} \quad-$ convective and radiative heat flux $\left[\mathrm{W} / \mathrm{m}^{2}\right]$

$R C$ - radiation-conduction parameter

$S \quad$ - radiation source term

$S^{*} \quad-$ dimensionless radiation source term

$T \quad-$ temperature $[\mathrm{K}]$

$T_{c}, T_{h} \quad-$ top wall cold and hot temperature $[\mathrm{K}]$

$U_{0} \quad-$ moving lid velocity $[\mathrm{m} / \mathrm{s}]$

$(u, v) \quad-\quad$ the $x$ - and $y$-velocity components $[\mathrm{m} / \mathrm{s}]$

$(U, V) \quad-$ dimensionless the $x$ - and $y$-velocity components

$(x, y) \quad-$ dimensional Cartesian coordinate $[\mathrm{m}]$

$(X, Y) \quad-$ dimensionless Cartesian coordinate

$\alpha \quad-$ thermal diffusivity $\left[\mathrm{m}^{2} / \mathrm{s}\right]$

$\beta \quad-$ extinction coefficient $\left[\mathrm{m}^{-1}\right]$, or thermal coefficient expansion $\left[\mathrm{k}^{-1}\right]$

$\varepsilon \quad-$ emissivity of wall

$\theta, \theta_{1}, \theta_{2}$ - dimensionless temperature and dimensionless temperature parameters

$\mu \quad-$ dynamic viscosity $\left[\mathrm{N} \cdot \mathrm{s} / \mathrm{m}^{2}\right]$

$\nu \quad-$ kinematic viscosity $\left[\mathrm{m}^{2} / \mathrm{s}\right]$

$\rho \quad-$ density $\left[\mathrm{kg} / \mathrm{m}^{3}\right]$

$\sigma_{a}, \sigma_{s} \quad-$ absorbing and scattering coefficient $\left[\mathrm{m}^{-1}\right]$

$\tau \quad-$ optical thickness

$\Phi \quad-\quad$ scattering phase function

$\omega \quad-$ scattering albedo

$\Omega \quad-$ solid angle

and

$\left.\mathrm{Gr}=g \beta\left(T_{h}-T_{c}\right) L^{3}\right) / \nu^{2}, \operatorname{Pr}=\nu / \alpha, \operatorname{Re}=L U_{0} / \nu, \operatorname{Ri}=\mathrm{Gr} / \operatorname{Re}^{2}, \operatorname{Pe}-$ Grashof, Prandtl, Reynolds, Richardson and Peclet number, respectively

$\mathrm{Nu}, \mathrm{Nu}_{t}-$ Nusselt and total Nusselt number at bottom wall, respectively

$\mathrm{Nu}_{c}, \mathrm{Nu}_{r}$ - convective and radiative Nusselt number, respectively

\section{Mathematical model}

The schematic diagram of a lid-driven trapezoidal cavity is shown in Fig. 1. The top lid of the cavity moves in the $x$-direction with constant velocity $U_{0}$. The horizontal walls are isothermal and the temperature of bottom wall $T_{h}$ is higher than that of the moving wall $T_{c}$. Also, the side walls are assumed with linear temperature distribution in the range of $T_{c}<T<T_{h}$, and their inclination angle is equal to $45^{\circ}$. All thermo-physical properties of the fluid except the density are assumed to be constant. The fluid into the enclosure is considered as a gray participating medium and all walls are considered to be black. 


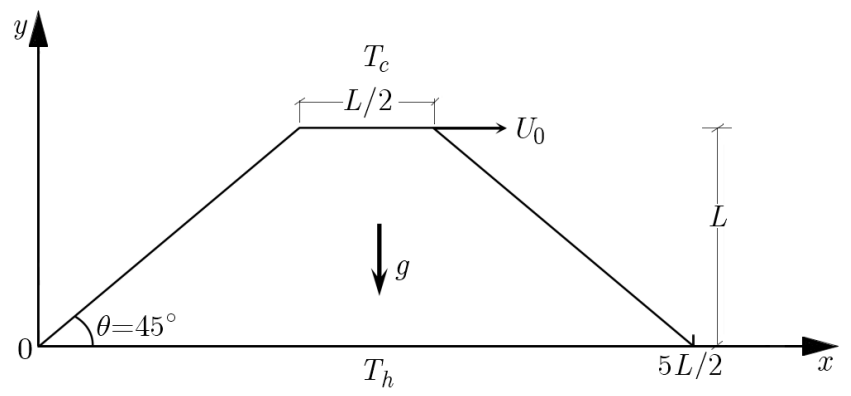

Fig. 1. Schematic model of the trapezoidal cavity

The governing equations containing continuity, momentum and energy for a two-dimensional, steady, laminar and constant property flow assuming the Boussinesq approximation in a dimensional form can be written as

$$
\begin{aligned}
& \frac{\partial u}{\partial x}+\frac{\partial v}{\partial y}=0 \\
& u \frac{\partial u}{\partial x}+v \frac{\partial u}{\partial y}=-\frac{1}{\rho} \frac{\partial p}{\partial x}+\vartheta\left(\frac{\partial^{2} u}{\partial x^{2}}+\frac{\partial^{2} u}{\partial y^{2}}\right) \\
& u \frac{\partial v}{\partial x}+v \frac{\partial v}{\partial y}=-\frac{1}{\rho} \frac{\partial p}{\partial y}+\vartheta\left(\frac{\partial^{2} v}{\partial x^{2}}+\frac{\partial^{2} v}{\partial y^{2}}\right)+g \beta\left(T-T_{c}\right) \\
& u \frac{\partial T}{\partial x}+v \frac{\partial T}{\partial y}=\frac{k}{\rho C_{p}}\left(\frac{\partial^{2} T}{\partial x^{2}}+\frac{\partial^{2} T}{\partial y^{2}}\right)-\frac{1}{\rho C_{p}} \nabla \cdot \mathbf{q}_{r}
\end{aligned}
$$

The radiative source term in the energy equation which appears as divergence of radiative flux $\nabla \cdot \mathbf{q}_{r}$ is dependent on the local radiation intensities (Modest, 2003)

$$
\nabla \cdot \mathbf{q}_{r}=\sigma_{a}\left(4 \pi I_{b}(\mathbf{r})-\int_{4 \pi} I(\mathbf{r}, \mathbf{s}) d \Omega\right)
$$

The local radiation intensity is calculated by solving the radiative transfer equation. This equation for the participating medium in the direction vector $\mathbf{s}$ can be expressed as (Modest, 2003)

$$
(\mathbf{s} \cdot \nabla) I(\mathbf{r}, \mathbf{s})=-\beta I(\mathbf{r}, \mathbf{s})+\sigma_{a} I_{b}(\mathbf{r})+\frac{\sigma_{s}}{4 \pi} \int_{4 \pi} I(\mathbf{r}, \mathbf{s}) \Phi\left(\mathbf{s}, \mathbf{s}^{\prime}\right) d \Omega^{\prime}
$$

In this study, the phase function $\Phi\left(\Omega, \Omega^{\prime}\right)$ is equal to unity because of the assumption of isotropic scattering.

For diffusely reflecting walls in Eq. (2.3), the radiative boundary condition is

$$
I\left(\mathbf{r}_{w}, \mathbf{s}\right)=\varepsilon_{w} I_{b}\left(\mathbf{r}_{w}\right)+\frac{1-\varepsilon_{w}}{\pi} \int_{\mathbf{n}_{w} \cdot \mathbf{s}<0} I\left(\mathbf{r}_{w}, \mathbf{s}\right)\left|\mathbf{n}_{w} \cdot \mathbf{s}^{\prime}\right| d s^{\prime} \quad \mathbf{n}_{w} \cdot \mathbf{s}>0
$$

The discrete ordinates method is used to solve the equation of radiative transfer equation (Eq. (2.3)). In this method, Eq. (2.3) is replaced by a set of $n$ equations for a finite number of $n$ different directions $\mathbf{s}_{i}$, and each integral is replaced by a quadrature series of the following form

$$
\left(\mathbf{s}_{i} \cdot \nabla\right) I\left(\mathbf{r}, \mathbf{s}_{i}\right)=-\beta I\left(\mathbf{r}, \mathbf{s}_{i}\right)+\sigma_{a} I_{b}(\mathbf{r})+\frac{\sigma_{s}}{4 \pi} \sum_{j=1}^{n} w_{j} I\left(\mathbf{r}, \mathbf{s}_{j}\right) \Phi\left(\mathbf{s}_{j}, \mathbf{s}_{i}\right) \quad i=1,2,3, \ldots, n
$$

with the boundary conditions

$$
\left.I\left(\mathbf{r}_{w}, \mathbf{s}_{i}\right)=\varepsilon_{w} I_{b}\left(\mathbf{r}_{w}\right)+\frac{1-\varepsilon_{w}}{\pi} \sum_{\left|\mathbf{n}_{w}\right|,\left|\mathbf{s}_{j}\right|<0}\right) w_{j} I\left(\mathbf{r}_{w}, \mathbf{s}_{j}\right)\left|\mathbf{n}_{w}, \mathbf{s}_{j}\right|
$$


In the above equations, $w_{k}$ is the weighting factor for each discrete ordinate. The original equation by this angular approximation is transformed to a set of coupled differential equations. Equation (2.5) in Cartesian coordinates becomes

$$
\begin{aligned}
& \xi_{i} \frac{\partial I_{i}}{\partial x}+\nu_{i} \frac{\partial I_{i}}{\partial y}+\mu_{i} \frac{\partial I_{i}}{\partial z}+\beta I_{i}=\beta S_{i} \\
& S_{i}=(1-\omega) I_{b}+\frac{\omega}{4 \pi} \sum_{j=1}^{n} w_{j} I\left(\mathbf{r}, \mathbf{s}_{j}\right) \Phi\left(\mathbf{s}_{j}, \mathbf{s}_{i}\right) \quad i=1,2,3, \ldots, n
\end{aligned}
$$

in which $\xi_{m}, \nu_{m}, \mu_{m}$ are the directional cosines of $\mathbf{s}_{i}$.

Non-dimensional Eqs. (2.1)-(2.3) are obtained using the following dimensionless variables

$$
\begin{array}{llll}
X=\frac{x}{L} & Y=\frac{y}{L} & U=\frac{u}{U_{0}} & V=\frac{v}{U_{0}} \\
\theta=\frac{T-T_{c}}{T_{h}-T_{c}} & P=\frac{p}{\rho U_{0}^{2}} & \operatorname{Re}=\frac{\rho U_{0} L}{\mu} & \theta_{1}=\frac{T_{c}}{T_{h}-T_{c}} \\
\theta_{2}=\frac{T_{h}}{T_{c}} & I^{*}=\frac{I}{\sigma T_{h}^{4}} & S^{*}=\frac{S}{\sigma T_{h}^{4}} & \tau=\beta L \\
1-\omega=\frac{\sigma_{a}}{\beta} & \operatorname{Pr}=\frac{\vartheta}{\alpha} & \operatorname{Pe}=\operatorname{Re} \operatorname{Pr} & \mathrm{Gr}=\frac{g \beta\left(T_{h}-T_{c}\right) L^{3}}{\vartheta^{2}} \\
R C=\frac{\sigma T_{h}^{3} L}{k} & q_{r}^{*}=\frac{q_{r}}{\sigma T_{h}^{4}} &
\end{array}
$$

The non-dimensional form of Eqs. (2.1)-(2.3) can be written as

$$
\begin{aligned}
& \frac{\partial U}{\partial X}+\frac{\partial V}{\partial Y}=0 \\
& U \frac{\partial U}{\partial X}+V \frac{\partial U}{\partial Y}=-\frac{\partial P}{\partial X}+\frac{1}{\operatorname{Re}}\left(\frac{\partial^{2} U}{\partial X^{2}}+\frac{\partial^{2} U}{\partial Y^{2}}\right) \\
& U \frac{\partial V}{\partial X}+V \frac{\partial V}{\partial Y}=-\frac{\partial P}{\partial Y}+\frac{1}{\operatorname{Re}}\left(\frac{\partial^{2} V}{\partial X^{2}}+\frac{\partial^{2} V}{\partial Y^{2}}\right)+\frac{\mathrm{Gr}}{\operatorname{Re}^{2}} \theta \\
& \frac{\partial}{\partial X}\left(U \theta-\frac{1}{\operatorname{Pe}} \frac{\partial \theta}{\partial X}\right)+\frac{\partial}{\partial Y}\left(V \theta-\frac{1}{\operatorname{Pe}} \frac{\partial \theta}{\partial Y}\right)+\frac{\tau(1-\omega) R C \theta_{1} \theta_{2}}{\operatorname{Pe}}\left[\frac{4}{\theta_{2}^{4}}\left(\frac{\theta}{\theta_{1}}+1\right)^{4}-\sum_{i=1}^{n} I_{i}^{*} w_{i}\right]=0
\end{aligned}
$$

The modified boundary conditions are:

- on the upper wall

$$
U=1 \quad V=0 \quad \theta=0
$$

— on the lower wall

$$
U=0 \quad V=0 \quad \theta=1
$$

— on the inclined walls

$$
U=0 \quad V=0 \quad \theta=1-Y
$$

The convective, radiative and total Nusselt number at the walls are determined using the heat fluxes as

$$
\mathrm{Nu}_{c}=\frac{q_{c} L}{k \Delta T} \quad \mathrm{Nu}_{r}=\frac{q_{r} L}{k \Delta T} \quad \mathrm{Nu}_{t}=\mathrm{Nu}_{c}+\mathrm{Nu}_{r}
$$




\section{Numerical solution procedure}

The applied numerical method to solve the governing equations (Eqs. (2.9)) is a line-by-line iteratively method based on the SIMPLE algorithm. The equations are discretized using the finite control volume technique on staggered control volumes for the $x$ and $y$ velocity components. The RTE is solved using the DOM method to calculate the radiation source term in the energy equation (Modest, 2003). This calculations are written in a FORTRAN program. The convergence criterion for solving the governing equations is assumed that the amount of residual terms between two consecutive iteration is less than $10^{-6}$, and the convergence criterion for the RTE is

$$
\max \left|\frac{I_{p}^{* n}-I_{p}^{* n-1}}{I_{p}^{* n}}\right| \leqslant 10^{-5}
$$

\section{Validation of the code}

To validate the pure mixed convection case, comparison is performed with the studied problem by Bhattacharya et al. (2013). The schematic of this problem is a two-dimensional trapezoidal enclosure with cold top wall (lid), hot bottom wall and adiabatic inclined walls. The distribution of the Nusselt number at the bottom wall for $\operatorname{Re}=100, \mathrm{Gr}=10^{5}$ and $\operatorname{Pr}=0.7$ is shown in Fig. 2. We can conclude that there is a good consistency between the present numerical results and those reported by Bhattacharya et al. (2013).

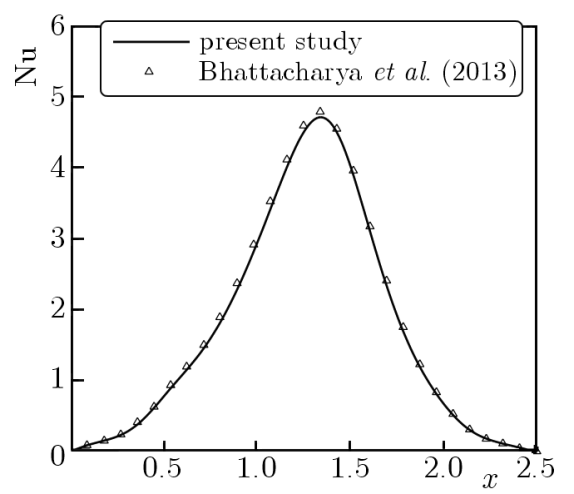

Fig. 2. Nusselt number profile at the bottom wall, $R C=10, \operatorname{Re}=100, \mathrm{Gr}=10^{5}$ and $\operatorname{Pr}=0.7$

To investigate validity of the discrete ordinates method, since no study exists on the combined heat transfer of mixed convection and radiation in a lid-driven cavity, a combined conductiveradiative heat transfer problem is considered. This problem was studied by Mahapatra et al. (2006). The schematic is a square enclosure of length $L$, containing an absorbing, emitting, and scattering medium. A plot of the mid-plane temperature inside the medium for $R C=10$, $\varepsilon=1.0, \tau=1.0$ and $\omega=0.5$ is shown in Fig. 3. It is shown that the present numerical results are in accordance with the theoretical ones reported by Mahapatra et al. (2006).

Also, to get grid-independent solutions, the maximum total Nusselt number at the bottom wall for three different radiation-conduction parameters and optical thicknesses for three cases are shown in Table 1. As it is seen, the amount of the total Nusselt number is different only in the second or third decimal places. Therefore, we used a uniform mesh with $90 \times 80$ grid numbers for all cases.

Also, the maximum total Nusselt number at the bottom wall with $S 4$ and $S 6$ approximations at $\operatorname{Re}=100, \mathrm{Gr}=10^{5}, \mathrm{Pr}=0.7$ is shown in Table 2 . These results demonstrate that there is very little difference between $S 4$ and $S 6$ approximations. Thus, to reduce computational effort, $S 4$ approximation is used for all cases. 


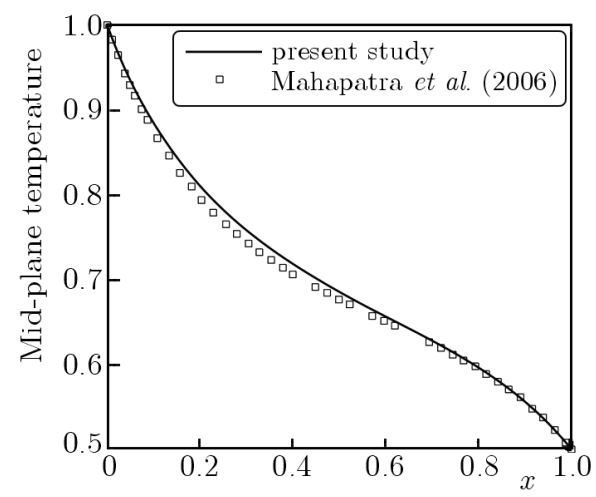

Fig. 3. Variation of mid-plane temperature, $\varepsilon=1.0$ and $\tau=1.0$

Table 1. Grid independence study in terms of the total Nusselt number at $\operatorname{Re}=100, \mathrm{Gr}=10^{5}$ and $\operatorname{Pr}=0.7$

\begin{tabular}{|c|c|c|c|c|c|c|}
\hline Grid Size & $\tau=0$ & $\tau=0.5$ & $\tau=1$ & $R C=0.5$ & $R C=2$ & $R C=5$ \\
\hline \hline $80 \times 70$ & 13.325 & 11.799 & 10.927 & 7.169 & 2.432 & 13.916 \\
\hline $90 \times 80$ & 13.310 & 11.781 & 10.916 & 7.157 & 9.418 & 13.903 \\
\hline $100 \times 90$ & 13.313 & 11.781 & 10.907 & 7.148 & 9.408 & 13.895 \\
\hline
\end{tabular}

Table 2. Angular quadrature study in terms of the total Nusselt number at $\operatorname{Re}=100, \mathrm{Gr}=10^{5}$ and $\operatorname{Pr}=0.7$

\begin{tabular}{|c|c|c|c|c|c|c|}
\hline & $\tau=0$ & $\tau=0.5$ & $\tau=1$ & $R C=0.5$ & $R C=2$ & $R C=5$ \\
\hline \hline$S 4$ & 13.310 & 11.781 & 10.916 & 7.157 & 9.418 & 13.903 \\
\hline$S 6$ & 13.380 & 11.836 & 10.944 & 7.160 & 9.436 & 13.951 \\
\hline
\end{tabular}

\section{Results and discussion}

In this study, the effect of radiative heat transfer on the heat transfer and flow characteristics of the mixed convection in a lid-driven trapezoidal cavity is investigated numerically. Figures 4 and 5 indicate the isotherms and streamlines at $\mathrm{Ri}=10$ for different optical thicknesses and also for the pure mixed convection case. Optical thickness $\tau=0$ shows the results for a radiatively transparent medium. These figures demonstrate the sweep behavior on the isotherms and streamlines. An increase in the optical thickness from $\tau=0$ to $\tau=2$ makes the difference between isotherms for the pure mixed convection and the radiative case increases as well. But when the optical thickness increases from $\tau=2$ to $\tau=100$, the isotherms of pure mixed convection case will be obtained. In fact, for high values of optical thickness, the radiation intensity cannot permeate the cavity, and it is absorbed near the walls.

This phenomenon is also seen for the streamlines along the optical thickness, such that by increasing the optical thickness from $\tau=0$ to $\tau=10$ the difference between streamlines for the pure mixed convection and the radiative case increases. But by further increasing of the optical thickness the reverse behavior is observed.

In Fig. 6, the effect of radiation-conduction parameter $(R C)$ on the thermal behavior of the system at $\mathrm{Ri}=10$ is shown. This parameter shows the relative importance of the radiative heat transfer mechanism compared with its conduction counterpart. As can be seen in Fig. 6, as the $R C$ parameter increases due to changing the dominant heat transfer mechanism from conduction to radiation, the difference between the isotherms for the radiative and pure mixed convection case increases. 

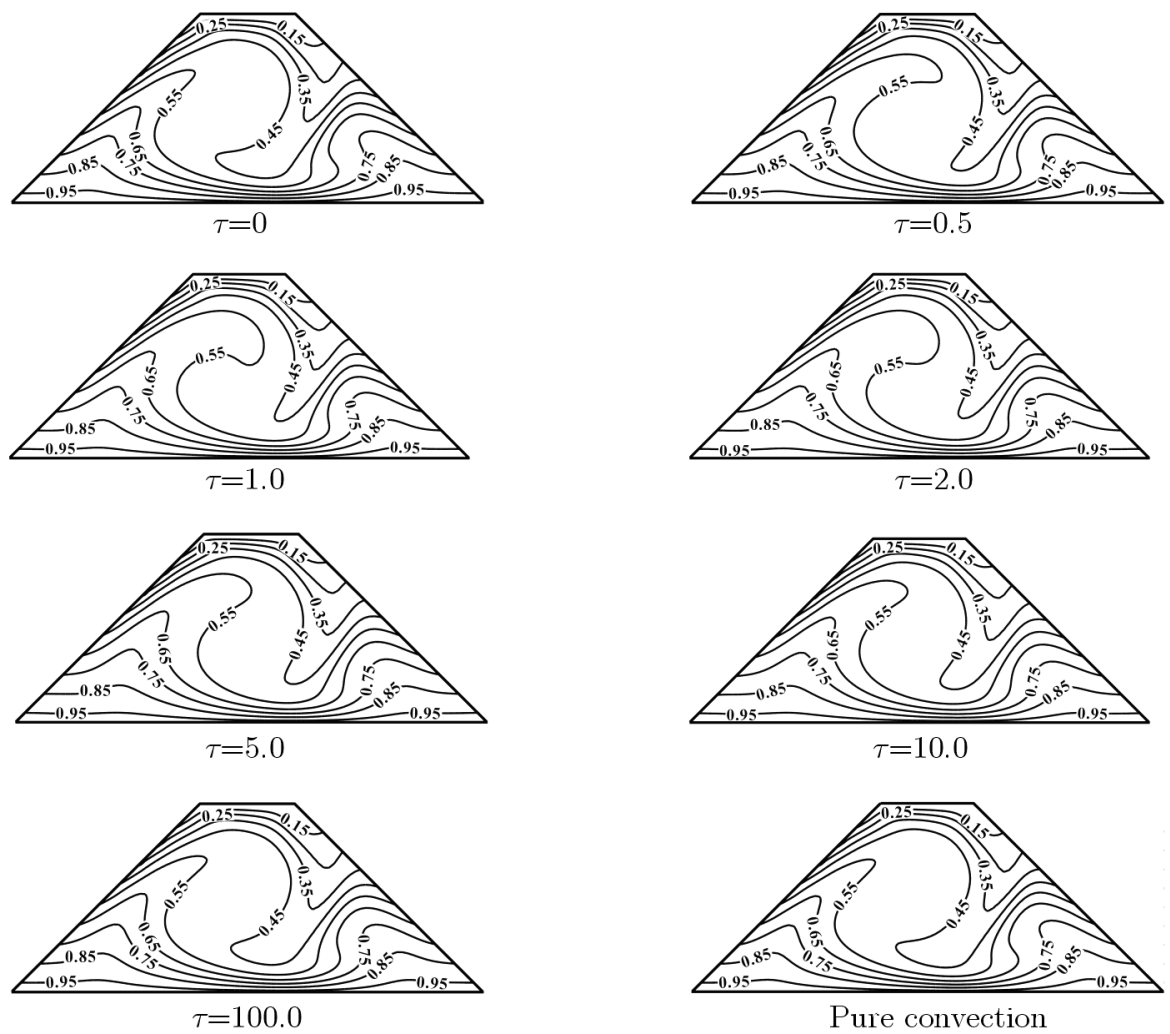

Fig. 4. Isotherms for different optical thicknesses and the pure mixed convection case at $\mathrm{Ri}=10$
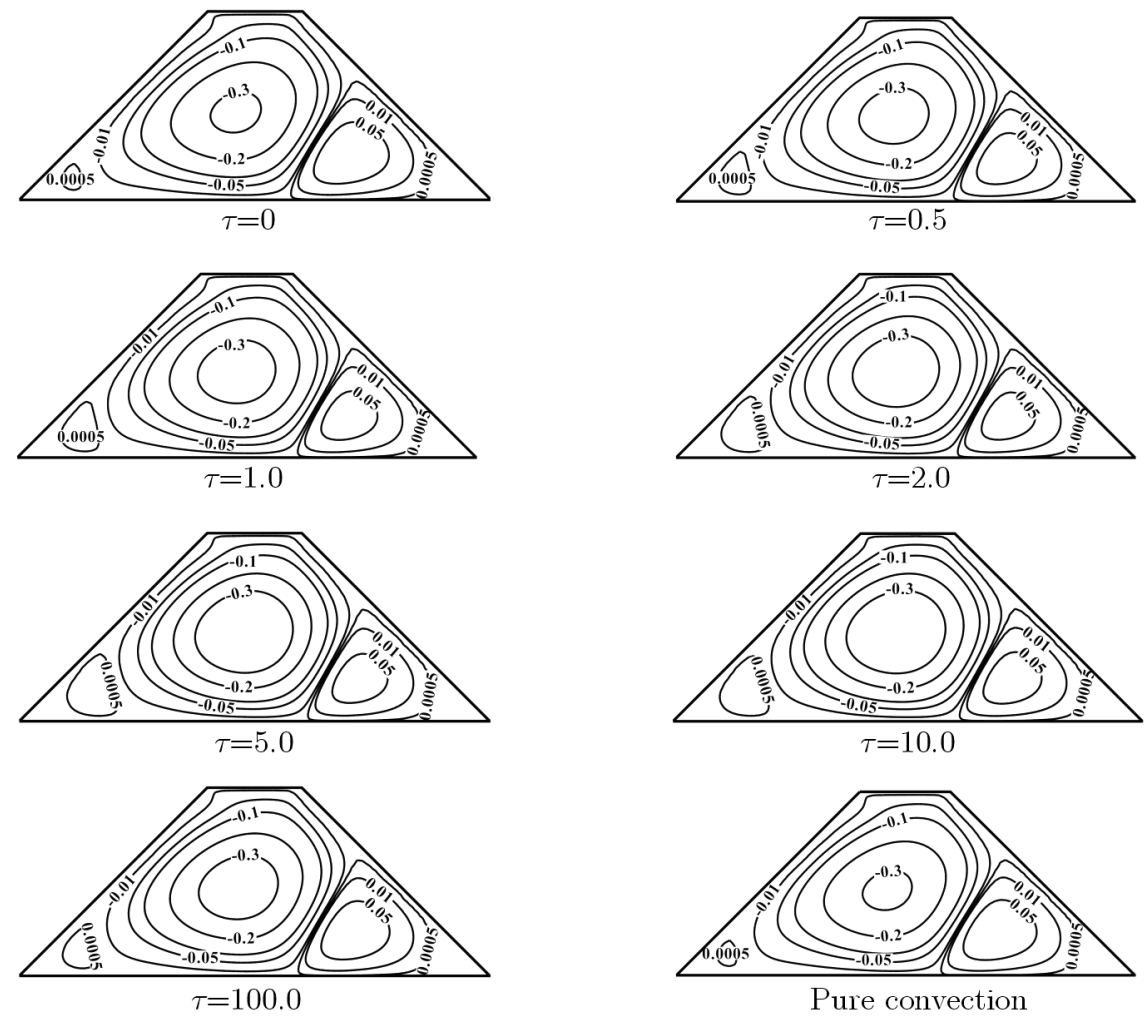

Fig. 5. Streamlines for different optical thicknesses and pure mixed convection at $\mathrm{Ri}=10$ 

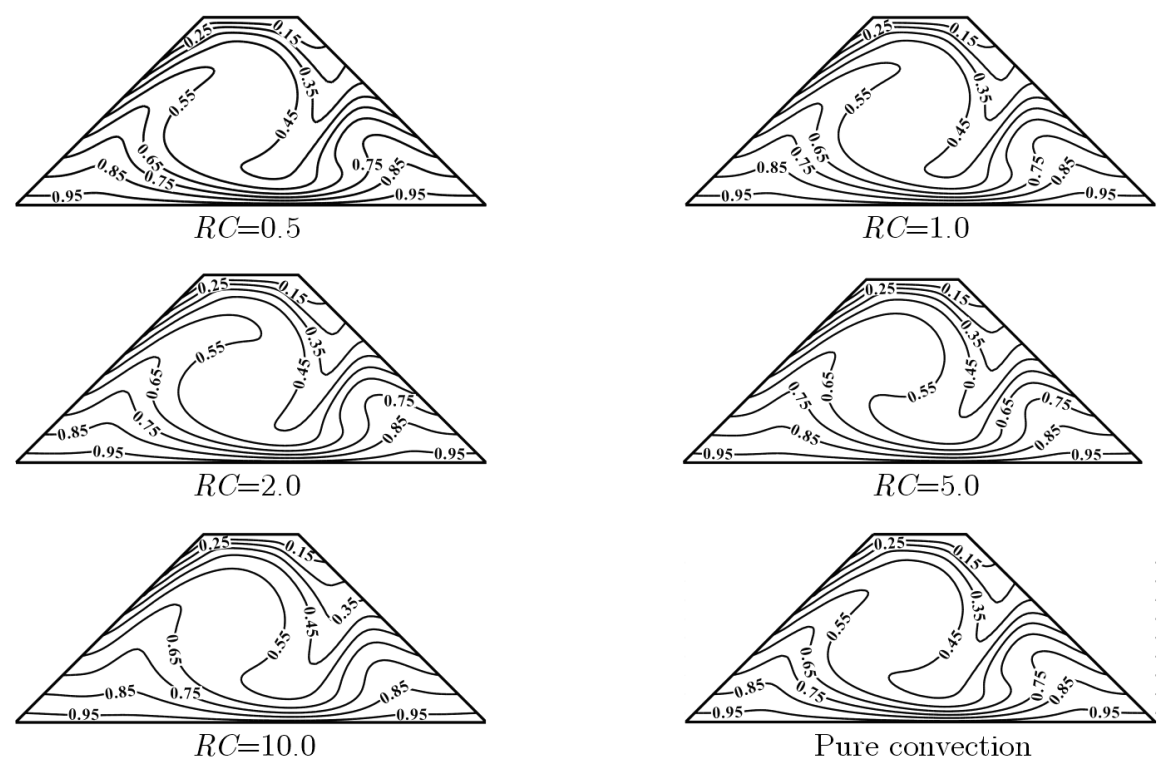

Fig. 6. Isotherms for different radiation-conduction parameters and pure mixed convection at $\mathrm{Ri}=10$

(a)

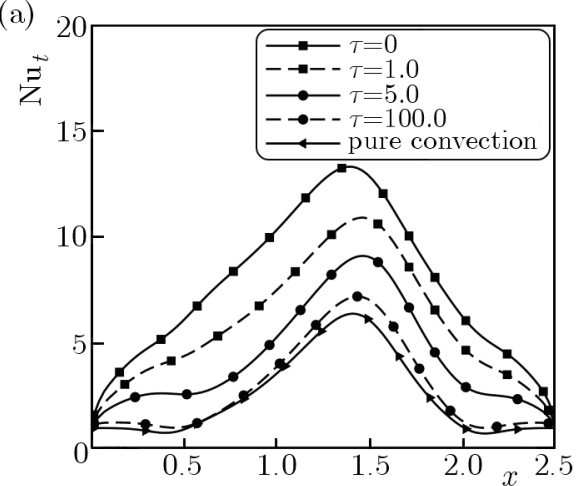

(b)

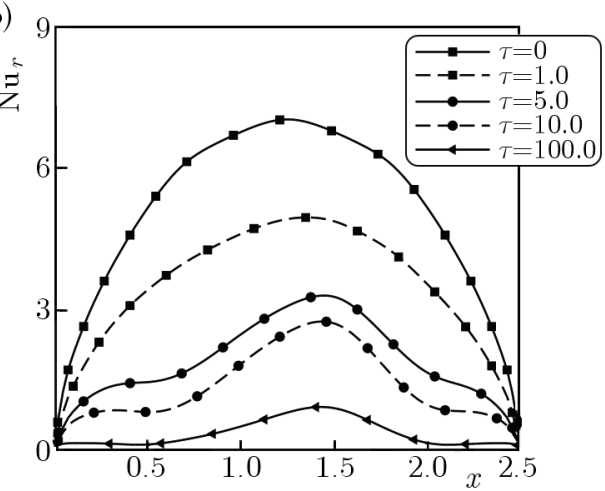

(c)

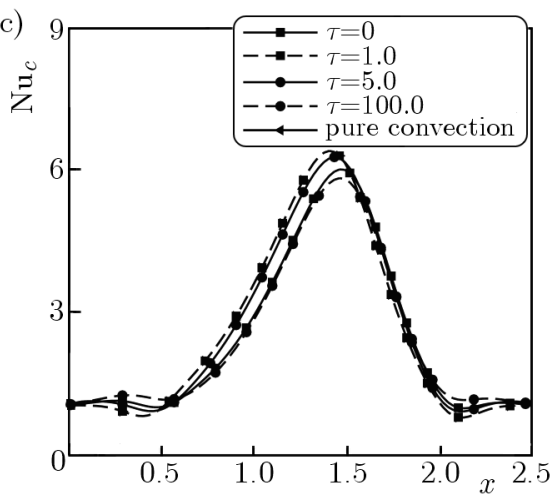

Fig. 7. The effect of optical thicknesses on the Nusselt number distribution along the bottom wall;

(a) total Nusselt number, (b) radiative Nusselt number, (c) convective Nusselt number

The radiative, convective and total Nusselt numbers along the bottom wall for different optical thicknesses at $\mathrm{Ri}=10$ are shown in Figs. 7a-7c. As it is seen in Fig. 7a, as optical thickness increases, the total Nusselt number decreases. This figure shows that the radiatively transparent medium $(\tau=0)$ has the maximum amount of the total Nusselt number and the pure mixed convection case has the smallest total Nusselt number. Therefore, it can be deducted that the radiatively transparent medium has the maximum amount of thermal exchange with the environment. Figure $7 \mathrm{~b}$ shows that as the optical thickness increases, the radiative Nusselt 
number decreases. This is because of the increasing gas absorption, hence the radiative heat flux at the bottom wall decreases. Figure $7 \mathrm{c}$ indicates that there is sweep behavior in the convective Nusselt number. By increasing the optical thickness from $\tau=0$ to $\tau=5$, the convective Nusselt number decreases at the core of the cavity $(0.5<X<1.5)$ and after that, with an increase in the optical thicknesses, the convective Nusselt number increases until it reaches the pure mixed convection, while near the inclined walls reverse behavior is observed on the convective Nusselt number with optical thickness.

Figures $8 \mathrm{a}-8 \mathrm{c}$ show the effect of the radiation-conduction parameter on the radiative, convective and total Nusselt numbers along the bottom wall at $\mathrm{Ri}=10$. Figures $8 \mathrm{a}$ and $8 \mathrm{~b}$ display that the total and radiative Nusselt numbers increase with an increase in the $R C$ parameter. But the convective Nusselt number decreases at the core of cavity $(0.5<X<1.5)$ with this parameter. This is because in high values of the $R C$ parameter, the radiative heat transfer is the dominant mechanism.
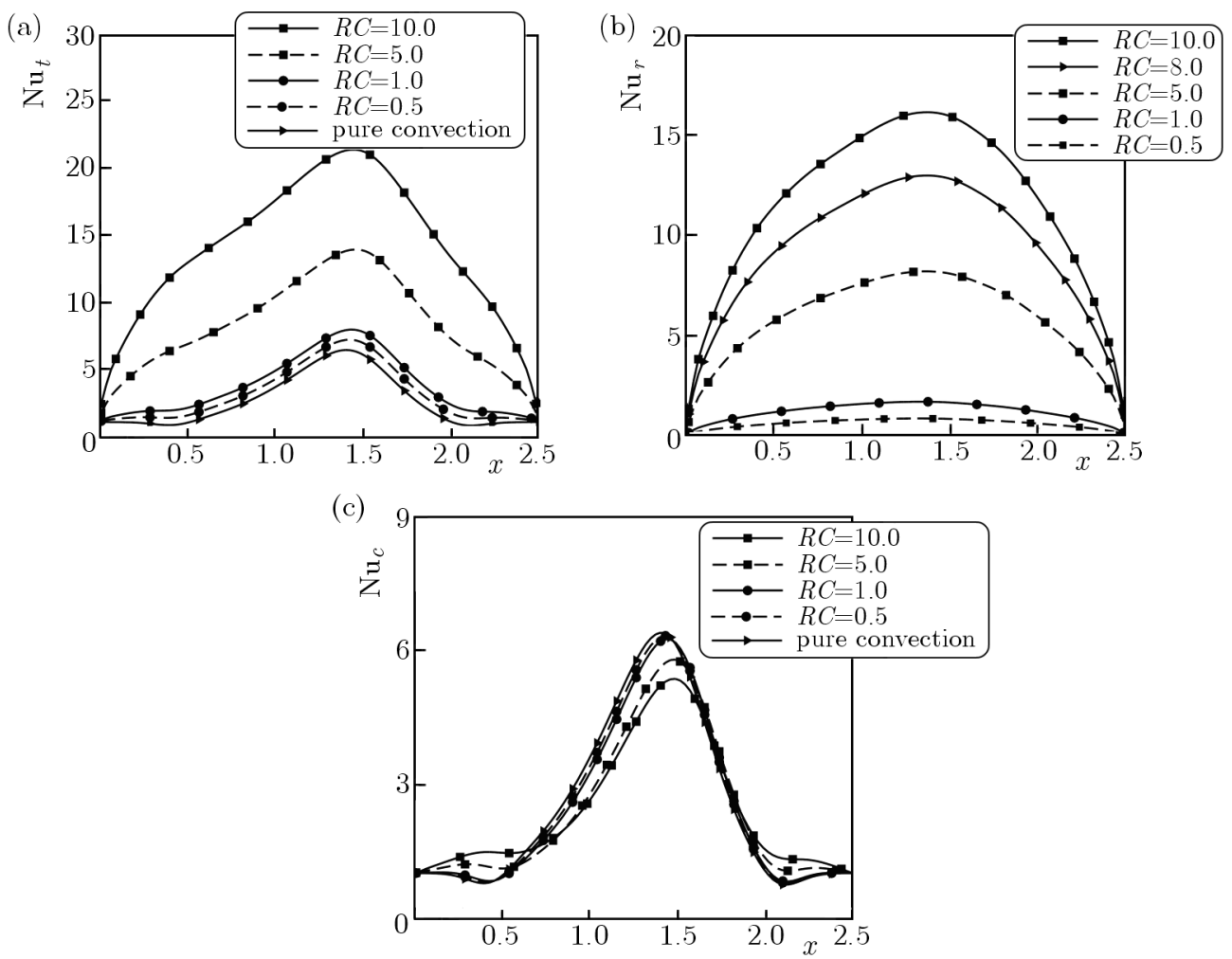

Fig. 8. The effect of radiation-conduction parameter on the Nusselt number distribution along the bottom wall; (a) total Nusselt number, (b) radiative Nusselt number, (c) convective Nusselt number

\section{Conclusions}

Mixed convection combined with radiation heat transfer in a lid-driven trapezoidal cavity has been analyzed numerically in the present study. The finite volume method has been chosen to solve the continuity, momentum and energy equations and the discrete ordinates method for the radiative transfer equation. The medium has been considered as emitting, absorbing and isotropically scattering with gray gases. The heat transfer and flow characteristics have been studied in the range of radiation-conduction parameter $(0.5 \leqslant R C \leqslant 10)$ and optical thickness $(0 \leqslant \tau \leqslant 100)$. The obtained results have shown that in a radiatively transparent medium, the radiative heat transfer is the dominant mechanism. It has the maximum heat transfer, whereas the pure convection case has the minimum heat transfer with the environment. Also, 
as the optical thickness increases, the contribution of radiation in comparison with convection decreases until pure mixed convection is obtained. But, by increasing the radiation-conduction parameter, the contribution of radiative heat transfer increases.

\section{References}

1. Bhattacharya M., Basak T., Oztop H.F., Varol Y., 2013, Mixed convection and role of multiple solutions in lid-driven trapezoidal enclosures, International Journal of Heat and Mass Transfer, 63, 366-388

2. Chen C.L., Cheng C.H., 2004, Experimental and numerical study of mixed convection and flow pattern in a lid-driven arc-shape cavity, Heat and Mass Transfer, 41, 58-66

3. Cheng C.H., Chen C.L., 2005, Numerical study of effects of inclination on buoyancy-induced flow oscillation in a lid-driven arc-shaped cavity, Numerical Heat Transfer Applications, 48, 77-97

4. Cheng T.S., LiU W.H., 2010, Effect of temperature gradient orientation on the characteristics of mixed convection flow in a lid-driven square cavity, Computers and Fluids, 39, 965-978

5. Mahapatra S.K., Dandapat B.K., Sarkar A., 2006, Analysis of combined conduction and radiation heat transfer in presence of participating medium by the development of hybrid method, Journal of Quantitative Spectroscopy and Radiative Transfer, 102, 277-292

6. Moallemi M.K., Jang K.S., 1992, Prandtl number effects on laminar mixed convection heattransfer in a lid-driven cavity, International Journal of Heat and Mass Transfer, 35, 1881-1892

7. Modest M.F., 2003, Radiative Heat Transfer, McGraw-Hill, New York

8. Oztop H.F., 2006, Combined convection heat transfer in a porous lid-driven enclosure due to heater with finite length, International Communications in Heat and Mass Transfer, 33, 772-779

9. Patankar S.V., Spalding D.B., 1972, A calculation procedure for heat, mass and momentum transfer in three-dimensional parabolic flows, International Journal of Heat and Mass Transfer, 15, 1787-1806

10. Prasad A.K., Koseff J.R., 1996, Combined forced and natural convection heat transfer in a deep lid driven cavity flow, International Journal of Heat and Fluid, 17, 460-467

11. Shankar P.N., Meleshko V.V., Nikiforovich E.I., 2002, Slow mixed convection in rectangular containers, Journal of Fluid Mechanics, 471, 203-217 